\title{
ARIDEZ Y NIEBLA EN LA COSTA DE TACNA
}

\author{
Leoncio Molina Vásquez ${ }^{\prime}$
}

\section{RES UMEN}

\begin{abstract}
El trabajo explica la formación de la niebla y aridez de Tacna como parte del fenómeno de la costa sur peruana, costa norchilena y altiplano boliviano, originado por la cercanía de la Cordillera de los Andes al mar y su geodinámica que, a su vez, ha hecho posible la formación de la fosa marina de Arica-Mollendo, por donde discurren las aguas frías de la corriente marina de Humboltd.
\end{abstract}

También explica desde el punto de vista termodinámico los procesos adiabáticos, seudoadiabáticos, isoentálpicos e isoentrópicos que se producen en la atmósfera de Tacna en condiciones isobáricas.

\section{A B S T R A C T}

In This work it is explained the formation of the fog and the aridity of Tacna like part of the phenomenon of the Peruvian south coast, norchilena coast and Bolivian highland, originated by the proximity of the Andes Mountains to the sea and its their geodinámica that, olso has made possible the formation of the marine grave of Arica-Mollendo, trough the cold waters of the marine current of Humboltd go.

It is also explained from the thermodynamic point of view the processes adiabaticos, seudoadiabáticos, isoentálpicos and isoentrópicos that take place in the atmosphere of Tacna in conditions isobáricas.

\section{INTRODUCCIÓN}

Muchas culturas tienen tanta relación directa con las condiciones meteorológicas que bien podrian denominarse "culturas del clima".

Los minerales depositados en climas frío y cálido son diferentes; los desiertos y lodos son el resultado de los procesos de lluvias, vientos intensivos y cambiantes donde destacan como testigos los radiolarios, que permiten los análisis de radioisótopos y físico químicos para determinar la edad de los depósitos terráqueos y cambio de clima.

Hoy se comienza a predecir los cambios de radiación solar, los factores metereológicos y climáticos por diferentes métodos, pero sin poder preveer aún en su verdadera magnitud el impacto en el medio ambiente de los cambios del clima por los fenómenos naturales, la lluvia ácida, el orificio en la capa de ozono, el efecto invernadero, aridización, etc.

\section{LA GEODINÁMICA Y LA TEORIA DE PLACAS}

La extensión del territorio sudamericano al Sur del Trópico, está sufriendo los efectos de una aridización natural iniciada con la regresión de los hielos del último periodo glaciar (pleistoceno inferior); y continúa hasta nuestros dias. La tierra como componente del Sistema Solar y con este el Sistema Galáctico, obedece a las leyes que gobiernan el Gran Sistema, que en su proceso de transformación y extinción buscan el equilibrio. Una de las manifestaciones de la tendencia al

1 Magister en Química. 
equilibrio final es el acondicionamiento de masas sólidas, líquidas y gaseosas por anomalías de los movimientos de rotación de la tierra, la luna y traslación del conjunto alrededor del sol.

Los últimos estudios han confirmado que la tierra tiene forma de pera. Eı polo norte se asemeja al tronco de un cono, mientras que el hemisferio sur tiene la forma de la parte inferior de la pera. El ensanchamiento en el trópico de Capricornio y el estrechamiento en el trópico de Cáncer, nos indican que la repartición de masas con respecto al eje de la tierra no es uniforme.

Todo ello, ha impuesto una geodinámica que tiende a la armonización de las fuerzas internas y externas de nuestro globo que, a su vez, se desplazan dentro de la dinámica cósmica.

Con los viajes cósmicos se están modificando y creando muchas teorias sobre los origenes y comportamiento de la tierra, como la Teoria Tectónica de Placas. Esta teoría se fundamenta en una serie de observaciones y mediciones de fenómenos ocurridos en el globo terráqueo. La geofísica y la geología se han puesto de acuerdo dando origen a la geodinámica. Para esta teoría, la litósfera de la tierra está dividida en placas que buscan el equilibrio, que es alterado por anomalías energéticas en los movimientos de la tierra, luna y sol. La litósfera está formada por los primeros 100 kilómetros de la envoltura de la tierra comprendiendo la corteza y parte superior de la tierra. En esta zona es donde ocurren todos los fenómenos físicos, geológicos y químicos que interesan a los seres vivientes. Según la "Teoría de Placas", la corteza de la tierra se divide en placas rígidas que se desplazan con movimientos horizontales, que en realidad son giros respecto al eje terráqueo. Los movimientos de placas son de deslizamiento cuando lo hacen en el mismo sentido; de extensión, cuando se aleja una de otra; de subducción, cuando una de las placas se desplaza debajo de la otra; como sucede cuando una placa marina empuja una placa continental dando lugar a los levamientos de sus bordes encontrados y tras ello la formación de dorsales marinos y cordilleras continentales.

Esta concepción satisface las observaciones y mediciones geofísicas y geológicas de la tierra, las ubicaciones de los epicentros de los sismos, la formación de las cordilleras, fosas marinas; la formación de las cordilleras y volcanes continentales, las grandes y pequeñas fallas, los volcanes nacientes, las anomalías de la gravedad, los movimientos de las grandes masas hídricas y gaseosas modificando los climas y las lluvias en la faz de la tierra.

\section{ASPECTO GENERAL DEL RELIEVE DE TACNA}

El área estudiada se extiende desde el nivel del mar hasta los 1,500 metros de altura en los flancos de la Cordillera Occidental de los Andes. En ella apreciamos cinco unidades fisiográficas diferentes, caracterizadas por la naturaleza de las formaciones infrayacentes y por sus rasgos propios de expresión topográfica.

El primer rasgo fisiográfico es el litoral: una angosta faja de terreno llano que se extiende entre la ribera del mar y el pie de la Cadena Costanera. Su ancho varía desde pocos metros en el extremo NO, Morro de Sama, hasta un máximo de $5 \mathrm{~km}$ a la altura de los cerros de La Yarada.

La ribera del mar, al Norte del río Sama, es sinuosa y presenta entrantes y numerosas puntas e islotes, tales como Comajuato, Isla Santa Rosa, Punta Mesa, Punta Panteón, Gentilar e Isla Vila Vila con pequeños desarrollos de playas como en Gentilar y Barredera Chica.

El litoral se encuentra desprovisto de vegetación y está cubierto por arenas eólicas.

Los cerros de la Cadena Costanera constituyen la segunda unidad fisiográfica. Consiste en una faja de terreno montañoso y accidentado con rumbo paralelo a la línea ribereña, y con una elevación máxima de 300 msnm en el sector del cerro Los Pabellones.

La tercera unidad fisiográfica se extiende entre la Cadena Costanera y el pie de los contrafuertes de los Andes; regionalmente se denominan "Pampas Costaneras" y consisten de un terreno llano, entre 200 y 1000 m de altitud. Su ancho varia entre 30 y 50 $\mathrm{km}$ y tiene una suave inclinación hacia el Sur y Suroeste. Esta unidad fisiográfica se extiende hasta el territorio chileno y se encuentra disectada por numerosas quebradas secas de cauce ancho y de poca profundidad. A la altura de la ciudad de Tacna ha sido fuertemente erosionada por el río Caplina.

El cono deyectivo del río Caplina constituye otra unidad fisiográfica. Se inicia en las cercanía de Punta Magollo y se ensancha progresivamente aguas abajo hasta alcanzar el Océano, adoptando una forma triangular cuya base mide aproximadamente $20 \mathrm{~km}$. 
El cono aluvial ha sido formado principalmente por el acarreo del río Caplina, con aporte de las quebradas Honda y Hospicio. La acción erosiva del río Caplina ha sido muy intensa durante el Cuaternario, habiendo cortado una diferencia de nivel de 340 entre la pampa del Alto de la Alianza y el piso del Valle.

En el borde oriental del cuadrángulo de Tacna, aproximadamente desde los 1000 metros de elevación, se destaca un rasgo de expresión topográfica particular al que se ha denominado Superficie de Huaylillas.

Es una Superficie caracterizada por un plano suavemente inclinado hacia el Sur y originada por la erosión de los tufos riolíticos blancos y blandos de la parte superior de la Formación Huaylillas; esta acción erosiva ha alcanzado los horizontes superiores de los tufos riolíticos rosados del miembro medio de dicha formación, que son característicos por su alto grado de compactación y dureza, y sobre el cual se ha formado la Superficie.

La Superficie de Huaylillas se extiende hacia el Norte y Este de Tacna, alcanzando su máximo desarrollo en el cuadrángulo de Huaylillas.

Se encuentra disectada por numerosas quebradas de cauces angostos y profundos, cuya orientación general sigue la dirección de la pendiente de la superficie, dando lugar a una topografía quebrada, caracterizada por la presencia de pequeñas planicies linitadas por barrancos.

Los ríos Caplina, Sama y Locumba son los cursos principales de drenaje del área estudiada; constituyen las únicas fuentes permanentes de agua que provienen de las precipitaciones pluviales y deshielos de los nevados situados en sus cuencas colectoras.

\section{TACNA Y LA ARIDIZACIÓN}

En América del Sur, la zona más afectada por la aridización es la comprendida entre los meridianos $65^{\circ}$ y $71,5^{\circ}$ de longitud Oeste y entre los paralelos $16^{\circ}$ y $28^{\circ}$ de latitud sur. Este casquete geodésico, comprende los territorios del Sur del Perú, norte de Chile y parte del altiplano boliviano, ubicado al Sur Este de la Cordillera Sur Occidental del Perú.

En esta zona nos interesa la Placa de Nazca que, por su ubicación en nuestras costas, ocasiona la aridización que se hace más notoria cada año. Esta placa ejerce presión contra el litoral del Pacífico desde Bio Bio (Chile) hasta el sur de Panamá; por el norte colinda con la placa Cocos y por el Sur es presionada por la placa Antártica (conformándose la elevación de la Cordillera Submarina Challenger), y por el Oeste colinda con la placa Pacífico (que da forma a la Cordillera del Pacífico).

Figura I. El Desierto de Atacama.

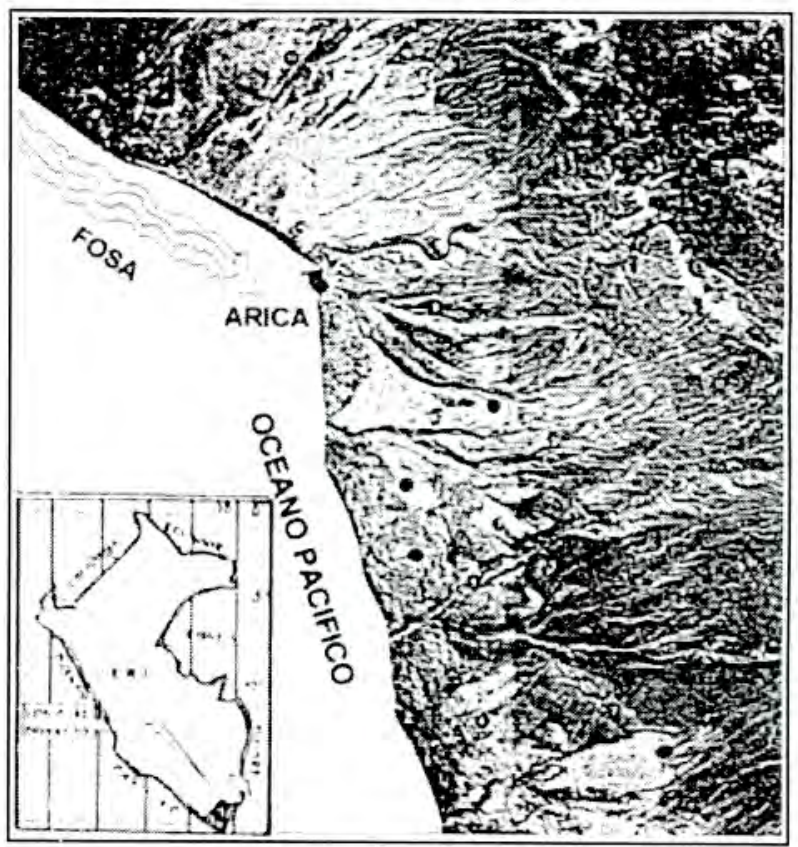

La Cordillera Challenger, el levantamiento del Pacífico y la dorsal de Nazca constituyen el marco de las elevaciones submarinas que conforman una gran tina, cuyo fondo más profundo es la fosa meridional que se extiende desde Arica hasta Mollendo, con una profundidad de 6867 metros frente a Arica y Tacna.

La corriente de la cuenca del Pacífico Sur-Este bordea la dorsal del Pacífico Antártico, de Oeste a Este, rebasando la elevación occidental de Chile e ingresando al litoral peruano; por la fosa marina, recorre este espacio lleno con agua fría, y continúa hacia el Norte, pasando por el cañón submarino que existe entre la dorsal de Nazca y tierra firme Sudamericana, sigue hacia el norte, donde la corriente de Humboldt se desplaza hacia la Isla de Galápagos y el mar cálido. La temperatura del agua de la corriente de Humboldt frente al litoral de Tacna y Moquegua varía desde los $13^{\circ} \mathrm{C}$ en la superficie a los $8^{\circ} \mathrm{C}$ a 500 metros y $5^{\circ} \mathrm{C}$ a $1000 \mathrm{~m}$ de profundidad.

Esta temperatura bajísima, de las aguas frente a Chile y el Sur del Perú, ocasionada por el gran depósito de agua fría, frente a sus litorales, hace que la evaporación marina esté limitada, de manera que en 
la atmósfera, no se alcance el grado de saturación, evitando que cuando las nubes choquen con un frente frío, se precipiten como lluvia por el crecimiento de las moléculas de agua y la gravedad.

Otro factor que limita fuertemente las precipitaciones en esta región son las barreras orográficas Cordillera Occidental de los Andes Perú-Chile y la Cordillera Oriental en Bolivia- que constituyen un frente frío que obligan a precipitaciones pluviosas del lado peruano, en el altiplano puneño y en Bolivia, en la región del Beni y de Santa Cruz de la Sierra.

\section{LA ARIDEZ DE LA PROVINCIA DESÉRTICA}

Una de las características de la provincia ecológica desértica es la aridez con una precipitación pluvial no mayor de $25 \mathrm{~mm}$ al año (Gráfico 1). A la costa de Tacna no le correspondería un clima desértico, según la latitud geográfica muy cercana a la línea ecuatorial; otras latitudes del mundo con latitudes geográficas semejantes presentan bosques tropicales.

Grafico 1. Precipitación en Tacna 1996-1997

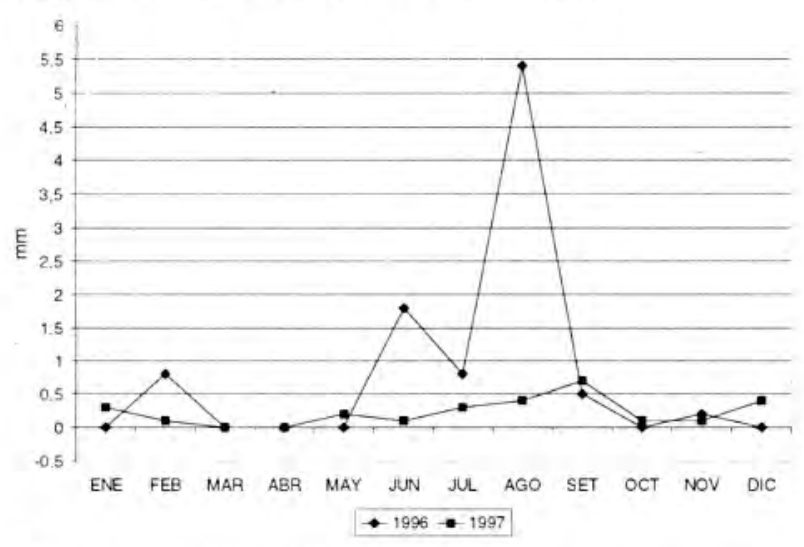

Fuente: SENAMHI-TACNA.

Las causas que determinan el carácter desértico de la costa sur peruana son:

La Cordillera de los Andes, situada muy cerca del Océano Pacífico, impide el paso de los vientos Alisios húmedos provenientes del Atlántico, produciendo intensas precipitaciones en la región de la Selva, cuyas estribaciones llegan al mar como ocurre con el Morro de Sama.

Los vientos Alisios, provenientes del Pacífico Sur, se dirigen hacia la costa, pierden humedad sobre el mar por la baja temperatura de las aguas de la corriente de Humboldt; producen una densa capa de neblina, y originan precipitaciones en forma de "garúa". A pesar de la alta humedad atmosférica (generalmente por sobre el $60 \%$ y llegando en la "época de neblina" hasta el $100 \%$ ) no caen lluvias debido al fenómeno de "inversión térmica" en la atmósfera.

Inversión térmica, es un fenómeno por el cual las aguas de la corriente de Humboldt enfrían la capa de aire situada encima de ellas hasta una altura de $800-900 \mathrm{msnm}$. Sobre la capa de neblina formada, la temperatura stube bruscamente de $13^{\circ} \mathrm{C}$ a $24^{\circ} \mathrm{C}$. Los vientos que soplan desde el mar, llevan esta neblina al interior de la Costa hasta una altura equivalente en el Continente, que impide el paso de los rayos solares (Tabla 1 )

Tabla 1. Dirección y velocidad del Viento ( $\mathrm{m} / \mathrm{seg}$.).

\begin{tabular}{|l|c|c|}
\hline \multicolumn{1}{|c|}{ MESES } & 1996 & 1997 \\
\hline Enero & $\mathrm{SSW} \mathrm{3}$ & $\mathrm{SSW} \mathrm{3}$ \\
\hline Febrero & $\mathrm{SSW} \mathrm{3}$ & $\mathrm{SSW} \mathrm{3}$ \\
\hline Marzo & $\mathrm{SSW} \mathrm{3}$ & $\mathrm{SSW} \mathrm{3}$ \\
\hline Abril & $\mathrm{SSW} \mathrm{3}$ & $\mathrm{SSW} \mathrm{3}$ \\
\hline Mayo & $\mathrm{SSW} \mathrm{3}$ & $\mathrm{SSW} \mathrm{2}$ \\
\hline Junio & $\mathrm{SSW} \mathrm{2}$ & $\mathrm{SSW} \mathrm{3}$ \\
\hline Julio & $\mathrm{SSW} \mathrm{2}$ & $\mathrm{SSW} \mathrm{2}$ \\
\hline Agosto & $\mathrm{SSW} \mathrm{3}$ & $\mathrm{SSW} 3$ \\
\hline Setiembre & $\mathrm{SSW} \mathrm{3}$ & $\mathrm{SSW} \mathrm{2}$ \\
\hline Octubre & $\mathrm{SSW} \mathrm{3}$ & $\mathrm{SSW} \mathrm{3}$ \\
\hline Noviembre & $\mathrm{SSW} \mathrm{3}$ & $\mathrm{SSW} \mathrm{3}$ \\
\hline Diciembre & $\mathrm{SSW} \mathrm{3}$ & $\mathrm{SSW} \mathrm{3}$ \\
\hline
\end{tabular}

Fuente: SENAMHI - TACNA.

Esta inversión térmica en las costas de Tacna impide la formación de lluvias; porque para producir una precipitación de agua en forma de "gotas", es necesario que las masas de aire húmedas sean llevadas a grandes alturas y por el enfriamiento del aire puedan formarse gotas. Esto no sucede en la "capa de nubes" en la costa de Tacna, dentro de los 800 - 900 msnm porque luego sigue el aire caliente; este aire absorbe toda la humedad de las nubes ascendentes e impide que estas lleguen a mayores alturas. El aire caliente llega hasta $2000-2500$ msnm en esta zona. En verano, cuando el calor del sol es intenso, desaparece "la capa de nubes". Las 
Iluvias que pueden caer en esta época son producidas por los vientos de las vertientes orientales, originadas por las nubes que pasan los Andes o el viento cálido que sube a la altura.

En los años de anomalías en el mar (gráfico 2), cuando se produce la corriente de EI Niño, se pueden producir lluvias en la costa, pero son insuficientes para revertir la aridización.

Grafico 2. Temperatura del Agua de Mar:

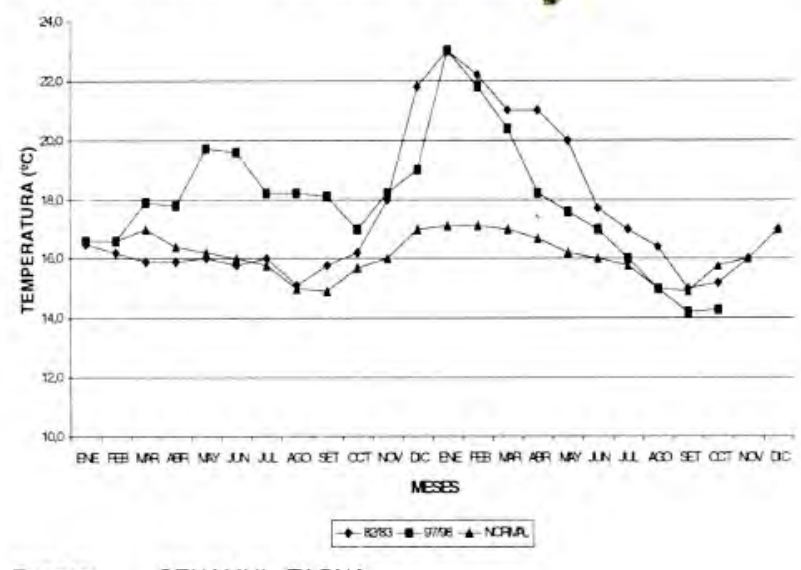

Fuente: SENAMHI - TACNA

\section{PROCESOS EN LA ATMOSFERA CON NEBLINA}

La explicación termodinámica del enfriamiento del aire húmedo por la presencia del aguas frías de la corriente de Humboldt está basada en procesos que producen enfriamiento isobárico, siendo:

$$
\mathrm{d} \mathrm{Q}=0 ; \mathrm{dP}=0 ; \mathrm{dh}=\mathrm{d} \mathrm{Q}
$$

Es decir, los procesos son adiabáticos. Dos de ellos además serán isobáricos, de modo que se cumplirá:

$$
\mathrm{d} \mathrm{Q}=0 ; \quad \mathrm{dP}=0 ; \quad \mathrm{dh}=\mathrm{Q}+\mathrm{VdP}=0
$$

Es decir, serán isentálpicos y en general no reversibles, como la evaporación y la condensación de agua en aire no saturado y la mezcla horizontal de dos masas de aire. La mezcla vertical de masas de aire puede considerarse como una combinación de dos procesos adiabáticos, uno isobárico y otro no.

De los dos procesos restantes, uno será adiabático reversible: la expansión de aire saturado, en forma reversible; este será por lo tanto un proceso isoentrópico. El último será realizado en un sistema abierto: expansión adiabática.
El hecho de que la mayor parte de los procesos, cuyo estudio resulta importante, en la atmósfera son adiabáticos, es fácil de comprender porque los sistemas considerados serán en general porciones más o menos grandes de la atmósfera, dejando de lado lo que ocurre en el contacto con el suelo y los enfriamientos por radiación. Los procesos de conducción de calor a través del aire son relativamente poco eficaces. Si la parcela aérea considerada es suficientemente grande, como para poder despreciar lo que ocurre en sus bordes, podremos tomarla como un sistema cerrado que no intercambia calor con el exterior. De acuerdo con la regla de fases, el aire húmedo tiene una varianza de 3 , mientras que una porción de nube (toda de agua) tiene varianza 2 . Las variables utilizadas serán $\mathrm{T}, \mathrm{P}$ y $\mathrm{W}$.

\section{FORMACIÓNDE LANEBLINA, ENFRIAMIENTO ISOBARICO}

Si una masa de aire se enfría a presión constante por debajo del punto de rocío, habrá en general condensación de agua. Este proceso exige la presencia de partículas que se constituyen en núcleos de condensación. De no existir estos no habría condensación (por lo menos en un cierto intervalo de temperatura por debajo del punto de rocío) y el aire estaria sobresaturado de vapor de agua. El aire atmosférico contiene, sin embargo, abundantes núcleos de condensación y el fenómeno de sobresaturación prácticamente no ocurre.

Si una masa de aire atmosférico se enfría isobáricamente hasta descender su temperatura por debajo del punto de rocío, se producirá la condensación en forma de gotas microscópicas formadas sobre los núcleos de condensación, constituyendo una niebla. Esto reproduce en la atmósfera el enfriamiento por radiación del propio aire; al formarse las gotitas que son prácticamente cuerpos negros en las longitudes de onda en que irradian, la capa aumenta y se favorece más el enfriamiento. También puede ocurrir la condensación cuando una masa de aire se traslada horizontalmente sobre el suelo hacia zonas más frías. Enfriándose en este caso por conducción de calor al suelo (nieblas de advección). En ambos casos, el enfriamiento es prácticamente isobárico; las variaciones de presión a lo largo del día son, en general, muy pequeños para que pueda producir el enfriamiento en la capa de aire inmediatamente superior. 
Conviene precisar que una vez iniciada la condensación, el enfriamiento procede más lentamente, porque en gran parte es compensado por el calor latente de condensación. Virtualmente, pone límite al enfriamiento muy próximo al punto de rocío, hecho que tiene importancia en el pronóstico de temperaturas mínimas. (gráfico 3)

Grafico 3. Temperaturas de la Costa de Tacna.

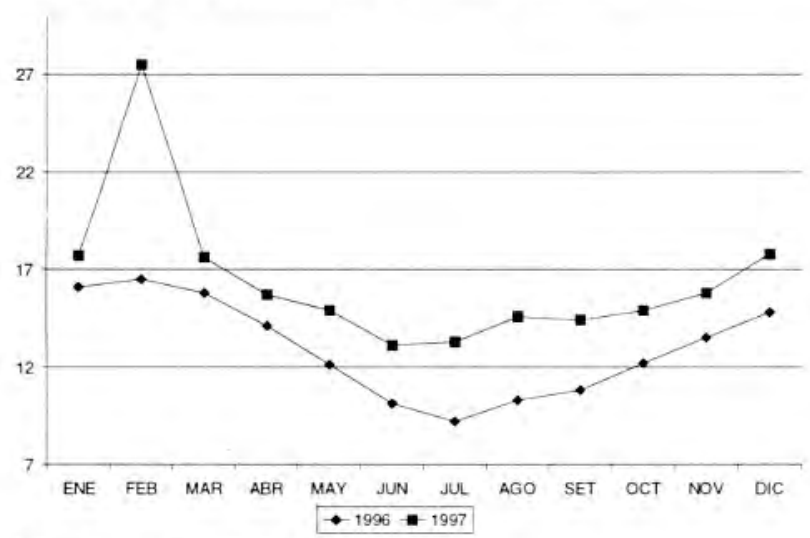

Fuente: SENAMHI - TACNA.

Cuando la temperatura baja del punto de rocío y la condensación es reducida, las gotitas formadas constituyen la neblina; sólo cuando la condensación se hace mayor se aplica el nombre de niebla. El pasaje es gradual y el límite es arbitrario. Este se establece por la visibilidad. Se dice así que hay niebla cuando - no se ven los objetos a más de un km de distancia, lo cual corresponde aproximadamente a una concentración de agua líquida en el aire de $\mathrm{H} 20=0,5 \mathrm{~g} /$ m3. El intervalo entre la saturación y la formación de esta cantidad de agua líquida se denomina intervalo de neblina y está marcado por la curva de saturación.

\section{MEZCLA ADIABATICA ISOBARICA (mezcla horizontal)}

El proceso adiabático isobárico (y por lo tanto isentálpico) de la mezcla de dos masas de aire húmedo, de distintas temperaturas y humedad, pero de igual presión en la atmósfera, corresponde a una mezcla horizontal entre la masa de aire húmedo dinámico y masa de aire húmedo estático en la atmósfera de Tacna.

Tendremos, designando las dos masas con sub índices 1 y 2 :

$$
\triangle \mathrm{H}=\mathrm{m}_{1} \quad \Delta \mathrm{h}_{1}+\mathrm{m}_{2} \quad \Delta \mathrm{h}_{2}=0
$$

Donde:

$$
\begin{aligned}
& \Delta \mathrm{h}_{1}-\mathrm{Cp}_{1}\left(\mathrm{~T}-\mathrm{T}_{1}\right) \quad(\mathrm{T}=\text { temperatura final }) \\
& \triangle \mathrm{h}_{2}=C \mathrm{p}_{2}\left(\mathrm{~T}-\mathrm{T}_{2}\right) \\
& C \mathrm{p}_{1}=\mathrm{C}_{\mathrm{pa}}\left(1+0,90 . \mathrm{q}_{\mathrm{i}}\right)
\end{aligned}
$$

\section{Sustituyendo $\mathrm{NH}$}

$$
\begin{aligned}
& \mathrm{m}_{1} C \mathrm{p}_{\mathrm{a}}(1+0,90)\left(\mathrm{q}_{\mathrm{i}}\right)\left(\mathrm{T}-\mathrm{T}_{1}\right)+\mathrm{m}_{2} \mathrm{C} \mathrm{p}_{2} \\
& (1+0,90)\left(\mathrm{q}_{2}\right)\left(\mathrm{T}-\mathrm{T}_{2}\right)=0
\end{aligned}
$$

\section{Despejando $T$}

$$
T=\frac{\left(m_{1} T_{1}+m_{2} T_{2}\right)+0,90\left(m_{1} q_{1} T_{1}+m_{2} q_{2} T_{2}\right)}{m+0,90\left(m_{1} q_{1}+m_{2} q_{2}\right)}
$$

\section{Masa total de vapor}

$$
\mathrm{m}_{\mathrm{v}}=\mathrm{m}_{1}+\mathrm{m}_{2}=\mathrm{m}_{1} \mathrm{q}_{1}+\mathrm{m}_{2} \mathrm{q}_{2}
$$

Si se desprecian los términos de vapor de agua, queda:

$$
\mathrm{T}=\frac{\mathrm{m}_{1} \mathrm{~T}_{1}+\mathrm{m}_{2} \mathrm{~T}_{2}}{\mathrm{~m}}
$$

La humedad específica final que se obtiene inmediatamente

$$
m q=\frac{m^{1 q_{1}}+m_{2} q_{2}}{m}
$$

Si se aplican las fórmulas aproximadas se obtiene la presión de vapor final

$$
P_{\text {vapor }}=\frac{m_{1} P_{1}+m_{2} P_{2}}{m}
$$

Hacemos notar que las últimas fórmulas valen para cualquier mezcla de dos masas de aire sin condensación, aunque las presiones sean distintas o varien, mientras que la última sólo vale para una mezcla isobárica, puesto que al deducirse se ha supuesto $P_{1}=P_{2}=P$.

\section{Niebla de Mezclas}

Según la carta higrométrica resulta claro que la mezcla de masa de aire húmedo produce un aumento de la humedad relativa. Como en el aire atmosférico no se mantienen sobre saturaciones apreciables, en el caso anterior habrá una condensación de vapor en 
gotas hasta llegar a la presión de vapor de saturación, que en las cartas se pueden representar por una recta correspondiente al proceso adiabático isobárico de condensación. En él se desprende calor latente, responsable del calentamiento de T a T'.

Se ve que este mecanismo es capaz, en principio, de producir nieblas de mezcla. Pero las condensaciones que se producen en la atmósfera de Tacna, no alcanzan a sobrepasar en general, el intervalo de la neblina.

\section{Expansión adiabática en la atmósfera}

Los procesos de expansión (o comprensión) adiabática son de particular importancia para analizar la neblina que se produce, porque describen las transformaciones que tienen lugar en una masa de aire que se eleva (o desciende) en la atmósfera. Es por lo tanto parte del estudio de la convección atmosférica. Consideraremos el ascenso de una parcela de aire que suponemos no se mezcla con su entorno, es decir, la expansión adiabática de un sistema cerrado constituído por aire húmedo. La primera etapa será una expansión adiabática húmeda de una fase gaseosa única. La adiabática húmeda, y el correspondiente descenso de temperatura, continuarán hasta que alcance a la temperatura de saturación, es decir, hasta que la temperatura de la humedad del aire es de saturación. A partir de ese momento, comienza la segunda etapa, en la que pueden ocurrir dos alternativas:

El agua que se va formando permanece en la parcela (nube), de tal modo que si descendiera, se produciría un proceso inverso a la comprensión adiabática, con evaporación de la fase condensada. En ese caso, se trata de una expansión adiabática saturada reversible (en realidad, para que el proceso se considere reversible, debe ocurrir con suficiente lentitud). Este proceso, por ser adiabático y reversible, es isoentrópico.

También puede ocurrir que el agua en formación esté saliendo simultáneamente y en forma total del sistema; en ese caso, el proceso no es reversible ni estrictamente adiabático, puesto que se trata de un sistema abierto que pierde masa. A este proceso se le llama seudo adiabático.

Es de suponer que en la atmósfera de Tacna ocurre muchas veces un proceso intermedio en que, a medida que el aire asciende, parte (no toda) del agua formada sale como lluvia o nieve.

\section{Proceso adiabático saturado reversible}

El sistema considerado para la explicación es una porción de nube que asciende, expandiéndose adiabática y reversiblemente; se trata de un sistema cerrado, que conserva toda el agua condensada. Por ser adiabático y reversible, el proceso también es isoentrópico.

\section{Donde la entropía es :}

$$
\begin{aligned}
\mathrm{S}= & \left(\mathrm{maC}_{\mathrm{pa}}+\mathrm{m}_{1} \cdot \mathrm{Cw}\right)(\ln \mathrm{T}-\mathrm{maRa} \ln \mathrm{Pa} \\
& +\underline{\operatorname{mvlv}(\mathrm{T})}+\text { Cons. }
\end{aligned}
$$

$\mathrm{T}$

\section{Donde :}

$$
\begin{aligned}
& \mathrm{Cw}=\text { calor específico del agua } \\
& \mathrm{I}=\text { calor molar específico de cambio fase }
\end{aligned}
$$

O bien, dividiendo por ma y considerando que la entropía debe mantenerse constante.

$$
\begin{aligned}
& \left(\mathrm{C}_{\mathrm{pa}}+\left(\mathrm{W}_{\mathrm{ts}} \cdot \mathrm{Cw}\right)(\ln \mathrm{T}-\mathrm{Raln} \mathrm{Pa}+\right. \\
& \frac{+\left(\mathrm{W}_{\mathrm{s}} \cdot \mathrm{P}_{\mathrm{v}}\right)}{\mathrm{T}}=\text { constante }
\end{aligned}
$$

\section{Donde :}

$$
\mathrm{Wts}=\frac{\mathrm{mt} ;}{\mathrm{ma}} \quad \mathrm{Ws}=\frac{\mathrm{mw}}{\mathrm{ma}} \quad \mathrm{Pa}=\mathrm{P}-\mathrm{ls}
$$

el sub índice s indica los valores correpondientes a la saturación.

\section{En forma diferencial queda :}

$$
\begin{aligned}
& \left(\mathrm{C}_{\mathrm{pa}}+\mathrm{W}_{\mathrm{t} 1 \mathrm{~s}} \cdot \mathrm{CW}\right) \mathrm{dR} \ln \mathrm{T}-\mathrm{Rad} \ln \mathrm{Pa}+ \\
& +\frac{\mathrm{d}\left(\mathrm{W}_{\mathrm{s}} \cdot \mathrm{lv}\right)}{\mathrm{T}}=0
\end{aligned}
$$

que describe el proceso adiabático saturado reversible.

\section{Proceso Seudo adiabático}

En este caso el agua condensada abandona inmediatamente el sistema. Se trata por lo tanto de 
un sistema abierto. Podemos, sin embargo, hallar la relación que nos interesa.

Dada la suposición hecha, debe ser en todo momento:

$$
\mathrm{m}_{\mathrm{w}}=0, \mathrm{~W}_{\mathrm{L} . \mathrm{s}}=\mathrm{W}_{\mathrm{s}}
$$

Además, podemos asignar al proceso de condensación de una cantidad dmw de agua y su alejamiento del sistema, como se efectuará en dos etapas.

Expansión adiabática saturada reversible, con condensación de las masa dmw de agua, y cuando el agua abandona el sistema.

En la primera etapa valdrá entonces:

$$
\begin{aligned}
& \left(\mathrm{C}_{\mathrm{pa}}+\mathrm{W}_{\mathrm{s}} \mathrm{C}_{\mathrm{w}}\right) \mathrm{d} \ln \mathrm{T}-\mathrm{Rad} \ln \mathrm{Pa}+ \\
& +\frac{\mathrm{d}\left(\mathrm{w}_{\mathrm{s}} \cdot \mathrm{Lv}\right)}{\mathrm{T}}=0
\end{aligned}
$$

En la segunda etapa la entropía del sistema disminuirá en el valor - Sw.dmw, pero no alterará los valores de $\mathrm{P}$ y $\mathrm{T}$ en cuanto a $\mathrm{Wt}, \mathrm{s}$ disminuya en:

Dmw

ma

De modo que se mantenga siempre igual a ws $(T)$. Por lo tanto, la ecuación (17) nos resuelve el problema de saber como varían $\mathrm{P}$ y $\mathrm{T}$ en un proceso seudo adiabático. Si se desea la forma integrada, será necesario tener en consideración que Ws depende de $\mathrm{T}$, a diferencia de Wt1s, así se tiene:

$$
\begin{aligned}
& \mathrm{C}_{\mathrm{pa}} \ln \mathrm{T}+\mathrm{C}_{\mathrm{w}} / \text { wsd } \ln \mathrm{T}-\mathrm{R} \text { aln } \mathrm{Pa}+ \\
& +\frac{\mathrm{W}_{\mathrm{s}} \cdot \mathrm{L}_{\mathrm{v}}}{\mathrm{T}}=\text { cte. }
\end{aligned}
$$

En la expansión seudo adiabática el enfriamiento es ligeramente más rápido que en el reversible.

\section{hemeroteCA CeNTRAL uhibG}

INADE/PELT. Microclimatología en el agrosistema de Waru Waru, Avance de Investigación Perú 1964.

MINISTERIO DE FOMENTO Y OBRAS. "Cuadrángulo La Yarada y Tacna - Boletín No.6 1963 - Perú.

MOSTACERO Y OTROS. Fundamento de Fitogeografia. Edit. Universidad Nacional de Trujillo. Perú 1990.

WARK WARNER. Contaminación del aire Edit. LIMUSA México 1990. 